\title{
Tracheal agenesis: rare but challenging
}

Volume 5 Issue 4 - 2016

Keywords: tracheal agenesis, neonates, pulmonary hypertension, fibroscopy

\section{Introduction}

Tracheal agenesis (TA) is a rare and severe congenital malformation of complete or almost complete failure of trachea development. ${ }^{1}$ Its prevalence is less than 1:50,000 with a male to female ratio of $2: 10^{2,3}$ It was initially described in 1900 by Payne and only few cases were published worldwide. ${ }^{1}$ It is associated with polyhydramnios and prematurity in more than half of the cases.

TA is classified by Floyd et al., ${ }^{4}$ into three anatomical subtypes: Type I, a short segment of the trachea fails to elongate upward to fuse with the larynx. Type II, the respiratory diverticulum fuses in the midline to form the carina but tracheal elongation does not take place. And type III, the respiratory diverticula does not fuse in the midline, resulting in two-fistula opening at the lower part of esophagus. ${ }^{4}$ Although type II TA is the most common form (up to 50$60 \%$ of cases), the prevalence of type II TA with bronchosophageal fistula is very rare. ${ }^{2}$ The etiology of this congenital anomaly is still unknown; Studies in mouse models suggested a role for the BMP type I receptor genes; however causal genes in human TA are yet to be identified. ${ }^{5}$ This case report depicts a Floyd's type II tracheal agenesis with bronchoesophageal fistula detected in the postnatal period in the absence of other congenital anomalies.

\section{Case report}

Our patient was a baby boy born term by normal vaginal delivery, without any apparent external abnormality (Weight: $3 \mathrm{Kg}$; Height: 50 $\mathrm{cm}$; Head circumference: $35.5 \mathrm{~cm}$ ), to a 27 year old mother G2 P1, non consanguineous parents, pregnancy complicated by polyhydramnios, with an APGAR score of 0 and 8 at 1 and 5 minutes respectively. He was intubated and ventilated (difficult intubation). However, he remained to have desaturations with serial arterial blood gases constantly showing $\mathrm{CO} 2$ retention (Arterial blood gas: $\mathrm{pH}=7.13$, $\mathrm{CO} 2=84, \mathrm{HCO} 3=26, \mathrm{pO} 2=95)$ despite adequate ventilator settings (CMV mode: $\mathrm{RR}=50, \mathrm{PIP}=18, \mathrm{PEEP}=3.5, \mathrm{FiO}=21 \%$ ).

At day three of life, patient was transferred to our hospital for further management. Patient was kept on CMV mode with persistence of his $\mathrm{CO} 2$ retention. Echocardiography done showed ASD Ostium Secundum $8 \mathrm{~mm}$ with left to right shunt and pulmonary hypertension ( $\mathrm{PAP}=30 \mathrm{mmHg}$ ). A bedside Fibroscopy was done revealing a blunted end suspecting tracheal agenesis. Computerized tomography scan (CT scan) confirmed the diagnosis of tracheal agenesis: the trachea extends for a distance of $1 \mathrm{~cm}$ after which it completely obliterates. A fistula connecting the larynx and the esophagus at the level of the vocal cords and another fistula separating the esophagus and the carina were noted (Figure 1).

These findings were further confirmed by direct laryngoscopic visualization in the operating room (Video). No surgical intervention was done due to the unstable and critical condition of the patient.

\section{Dany Hamod,' Sally El Helou,' Johnny Youssef,' Ziad Chebel,' Joseph Haddad,' Patrick Sneifer ${ }^{2}$ \\ 'Department of pediatrics, University of Balamand, Lebanon ${ }^{2}$ Department of Otolaryngology, University of Balamand, Lebanon}

Correspondence: Dany Hamod, Department of pediatrics, Division of Neonatology, Saint George Hospital University Medical Center, University of Balamand, Lebanon, Email dany_alhamod@hotmail.com

Received: September 19, 2016 | Published: October 18, 2016

Two days later, patient developed cardio respiratory arrest and was declared dead.

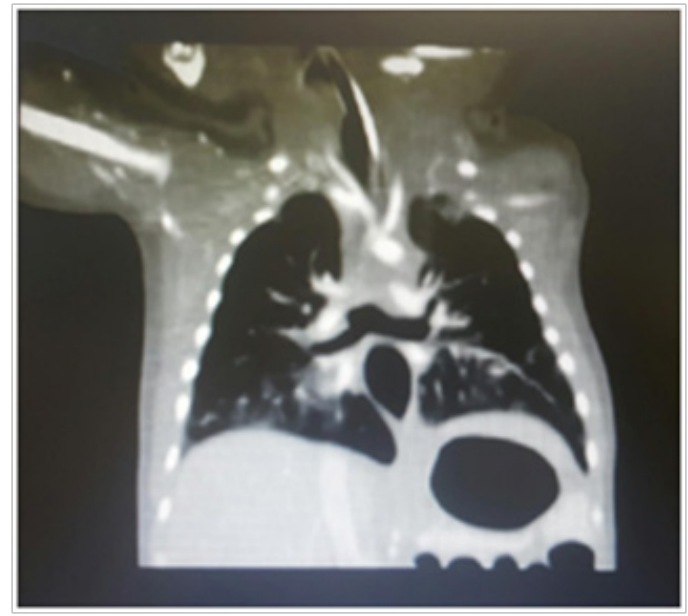

Figure I A fistula connecting the larynx and the esophagus at the level of the vocal cords and another fistula separating the esophagus and the carina were noted.

\section{Discussion}

Complete or almost complete failure of trachea development lead to tracheal agenesis. Less than 200 cases have been published. ${ }^{3}$ During the first eight weeks of gestations, the tracheo-pulmonary complex develops from the respiratory diverticulum at the ventral aspect of the primitive foregut. Vascular compromise has been postulated to be the cause of tracheal agenesis or tracheal stenosis. During development, the cephalic aspect of the respiratory diverticulum will elongate to form the trachea along with the infra-glottic structures. When this elongation process fails, it leads to tracheal agenesis. Usually it is associated with normal pulmonary and supra-glotic structures, as in our case. $^{6}$ 
Till now, no specific surgical intervention allows survival of patients with tracheal agenesis. A newborn with tracheal agenesis presents with immediate respiratory distress and an absent or very weak cry. Our case is compatible with type II TA, the most common abnormality (65\%), followed by type III $(22 \%)$ and type I (13\%). ${ }^{7}$

Tracheal agenesis is commonly associated with vertebral defects, anal atresia, tracheoesophageal fistula, esophageal atresia, cardiovascular defects, limb defects, duodenal atresia and renal defects (VACTERL) or cryptophtalmos, syndactyly, laryngeal atresia, and urogenital malformation (TARCD). ${ }^{2} \mathrm{~A}$ high index of suspicion is required for diagnosis and management due to the rare occurrence of this abnormal malformation. In the literature, up to $6 \%$ of type II TA present with absence of congenital anomaly. The presence of polyhydramnios antepartum raises the suspicion of tracheal agenesis. In our case, the presence of the bronchoesophageal fistula may have allowed the evacuation of the intrapulmonary liquid and hence the bilaterally enlarged echogenic lungs seen due to intrapulmonary liquid retention in congenital high airway obstruction syndrome (CHAOS) was absent prenatally. ${ }^{1,2}$

At birth, absent or a weak cry, acute respiratory distress with failure to intubate, increase oxygenation using face mask ventilation or placement of ET in the esophagus are factors that increase the suspicion of having tracheal agenesis. ${ }^{8}$ Bedside Fibroscopy, direct laryngo-esophagoscopy and CT scan can reveal the diagnosis. Prenatal diagnosis and early postnatal diagnosis with maintenance of airway patency at birth are essential for successful management of tracheal agenesis. Although prognosis is very poor, management of patients with TA is directed towards improving the quality of life and maintenance of patent airways allowing swallowing and speech. ${ }^{9}$

\section{Conclusion}

Tracheal agenesis, although a rare condition, should be always suspected in a newborn with immediate respiratory distress, weak or absent cry and failed intubation despite adequate ventilation with facemask. Endotracheal tube insertion in the esophagus with adequate ventilation will further increase the index of suspicion before the definitive diagnosis by Fibroscopy.

\section{Description of the video}

Direct laryngoscopy showed normal supraglottic anatomy. Passing between the vocal cords a blunt trachea $1 \mathrm{~cm}$ from the vocal cord associated with laryngotracheoesophageal cleft.

\section{Acknowledgments}

None.

\section{Conflicts of interest}

Authors declare that there is no conflict of interest.

\section{References}

1. Bertholdt C, Perdiolle-Galet E, Bach-Segura P, et al. Tracheal Agenesis: A Challenging Prenatal Diagnosis-Contribution of Fetal MRI. Case Reports in Obstetrics and Gynecology. 2015;(2015):3.

2. Ergun S, Tewfik T, Daniel S. Tracheal agenesis: A rare but fatal congenital anomaly. Mcqill J Med. 2011;13(1):10.

3. de Groot-van der Mooren MD, Haak MC, Lakeman P, et al. Tracheal agenesis: approach towards this severe diagnosis. Case report and review of the literature. Eur J Pediatr. 2012;171(3):425-431.

4. Floyd J, Campbell DC, Dominy DE. Agenesis of the trachea. American Review of Respiratory Disease. 1962;86(4):557-560.

5. Domyan ET, Ferretti E, Throckmorton K, et al. Signaling through BMP receptors promotes respiratory identity in the foregut via respiratory of Sox2. Development. 2011;138(5):971-981.

6. Ahmad R, Abdullah K, Mokhtar L, et al. Tracheal agenesis as a rare cause of difficult intubation in a newborn with respiratory distress: a case report. J Med Case Rep. 2009;3:105.

7. Fraser N, Stewart RJ, Grant J, et al. Tracheal agenesis with unique anatomy. J Pediatr Surg. 2005;40(10):e7-e10.

8. De Jose Maria B, Drudis R, Monclus E, et al. Management of tracheal agenesis. Paediatr Anaesth. 2000;10(4):441-444.

9. Hiyama E, Yokoyama T, Ichikawa T, et al. Surgical management of tracheal agenesis. J Thorac Cardiovasc Surg. 1994;108(5):830-833. 